\title{
Probability density tomography of microcavity polaritons confined in cylindrical traps of various sizes
}

\author{
Gaël Nardin*, Roland Cerna, Taofiq K. Paraïso, Barbara Pietka, Yoan Léger, \\ Ounsi El Daif ${ }^{1}$, François Morier-Genoud, Benoît Deveaud-Plédran
}

Laboratoire d'Optoélectronique Quantique, École Polytechnique Fédérale de Lausanne (EPFL), Station 3, CH-1015 Lausanne, Switzerland

\section{A R T I C L E I N F O}

\section{Article history:}

Available online 10 July 2009

\section{Keywords:}

Polaritons

Confined systems

Strong coupling

Semiconductors

\begin{abstract}
A B S T R A C T
We present the optical tomography of the probability density of microcavity polaritons, confined in three dimensions by cylindrical traps of various sizes. Collecting the photoluminescence emitted by the quasimodes under continuous nonresonant laser excitation, we reconstruct a three dimensional mapping of the photoluminescence, from which we can extract the spatial distribution of the confined states at any energy. We discuss the impact of the confinement shape and size on the probability density patterns.
\end{abstract}

(c) 2009 Elsevier Ltd. All rights reserved.

Microvavity polaritons are half-light half-matter quasiparticles, arising from the strong coupling between the electromagnetic mode of a semiconductor microcavity and a quantum well (QW) exciton [1]. The in-plane dispersion of the polaritons is dominated by their photonic component [2], and provides them with an effective mass $10^{4}$ smaller than the free electron mass. Microcavity polaritons can therefore be confined in the in-plane directions by traps with sizes in the micrometer range. While the imaging of carriers confined in semiconductor heterostructures is generally forbidden due to the small confinement size, probability density (PD) patterns of polaritons confined in micrometer size traps can be accessed by standard optical means [3,4].

We previously engineered a GaAs/AlAs microcavity with a single embedded InGaAs QW, featuring traps for the exciton-polaritons. These traps consist in quasi-circular mesas which were etched on the cavity spacer to locally enlarge the cavity length, thus providing a cylindrical confining potential for the photonic modes. More details about the sample and its fabrication are given in Ref. [5]. The strong

\footnotetext{
* Corresponding author. Tel.: +41216935449.

E-mail address: gael.nardin@epfl.ch (G. Nardin).

1 Present address: Institut des Nanotechnologies de Lyon (INL), UMR CNRS 5270, Ecole centrale de Lyon, 36 Avenue Guy de Collongue, 69134 Ecully Cedex, France.
} 


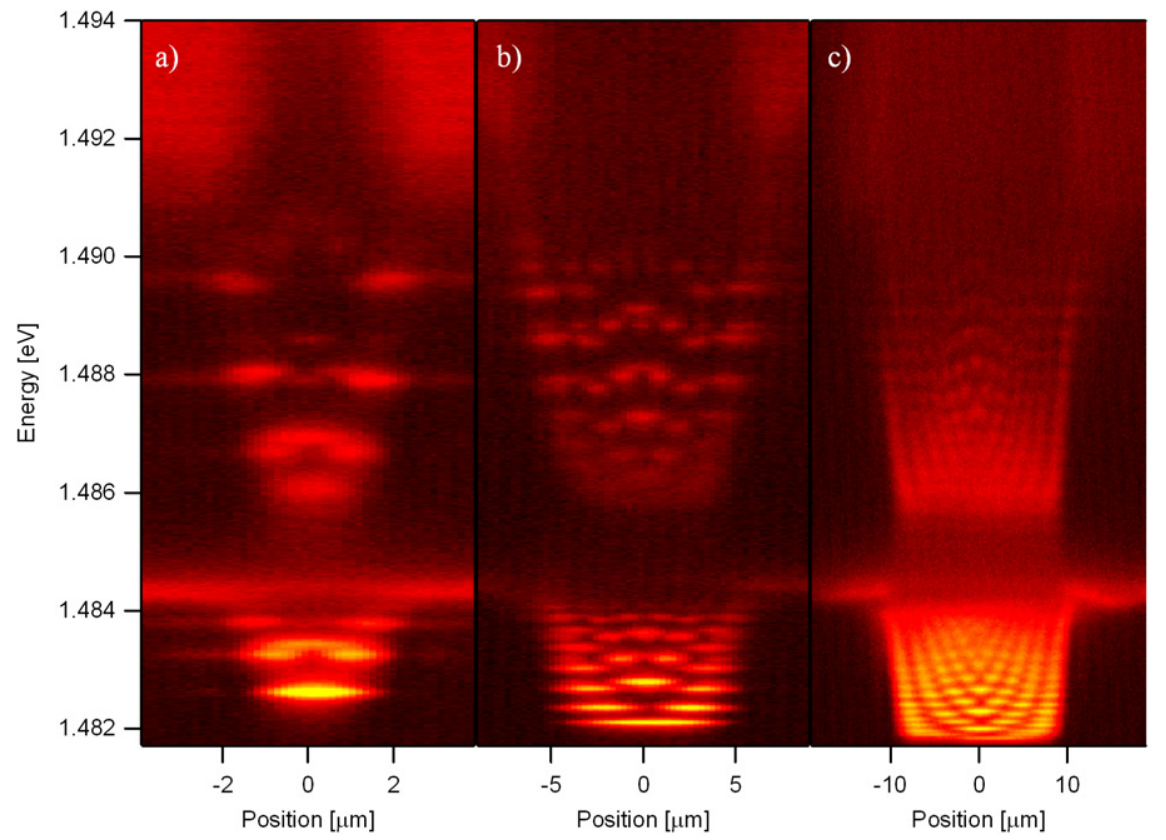

Fig. 1. Photoluminescence intensity as a function of position and energy, for a mesa of (a) $3.5 \mu \mathrm{m}$, (b) $9 \mu \mathrm{m}$ and (c) $19 \mu \mathrm{m}$ of diameter, in a $\log$ color scale. Position $x=0$ corresponds to the center of the mesa.

coupling between the confined photonic modes and the QW exciton gives rise to 0-dimensional (OD) exciton-polaritons [4-6].

We excite nonresonantly the system using a Ti:Sapphire cw laser focused on the sample. The diameter of the excitation spot is $30 \mu \mathrm{m}$. This hot excitation creates electron hole pairs which relax to the confined and delocalized polariton states $[7,8]$. Then, due to the coupling with extra-cavity photons, the eigenmodes emit photoluminescence (PL), which is collected with a 0.5 NA microscope objective and imaged on the slit of a spectrometer using a lens with $25 \mathrm{~cm}$ focal length, with a diffraction limited spatial resolution. At the output of the spectrometer, a liquid nitrogen cooled CCD camera records images of the PL with one spectral dimension and one spatial dimension. These spectra are presented in Fig. 1, for three different mesa diameters: $3.5 \mu \mathrm{m}$ (a), $9 \mu \mathrm{m}$ (b) and $19 \mu \mathrm{m}$ (c).

Fig. 1(a) shows the PL spectra of the upper and the lower polariton modes confined in a $3.5 \mu \mathrm{m}$ mesa, as well as the unconfined delocalized 2-dimensional (2D) modes that are still present around the mesa. Fig. 1(b) shows the spectrum of a $9 \mu \mathrm{m}$ mesa. Due to the larger confinement diameter, there are more confined states, for both the upper and lower polariton states, and the energy spacing between them is smaller. Fig. 1(c) shows the PL spectrum obtained for a $19 \mu \mathrm{m}$ diameter mesa. Even more confined states can be observed here. For the three confinement sizes, one can already observe the spatial distribution of the PD $\|\psi(r)\|^{2}$ of all the eigenstates along one axis.

We label the confined modes with quantum numbers $n$ and $m$ [9], $n=1,2,3, \ldots$ giving the number of lobes along the radial direction, $|2 m|$ (with $m=0, \pm 1, \pm 2, \ldots$ ) giving the number of nodes along the azimuthal direction.

The variations of emission intensity between the different confined states in Fig. 1(a)-(c) are due to the different photonic fraction of the confined states [10] and to thermalisation towards the lower energy states [7]. Ref. [7] shows as well that relaxation mechanisms and thermalisation are enhanced as the trap size decreases.

In order to perform a full tomography of the system, i.e. to obtain a 2D mapping of the PL at all energies in the same set of data, we shifted the position of the imaging lens perpendicularly to the optical axis. By this way we scanned the collected PL across the slit of the spectrometer, thus providing 
a

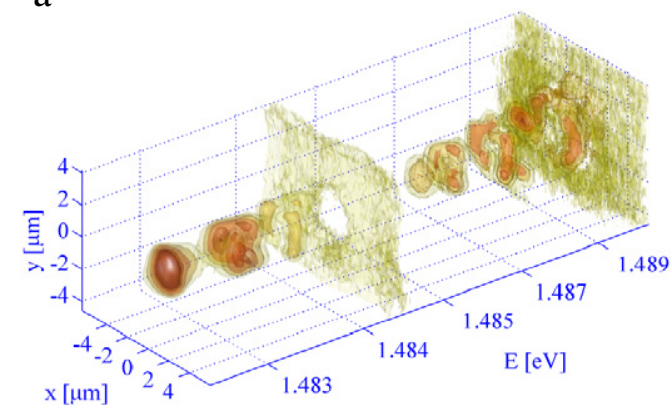

$\mathrm{b}$

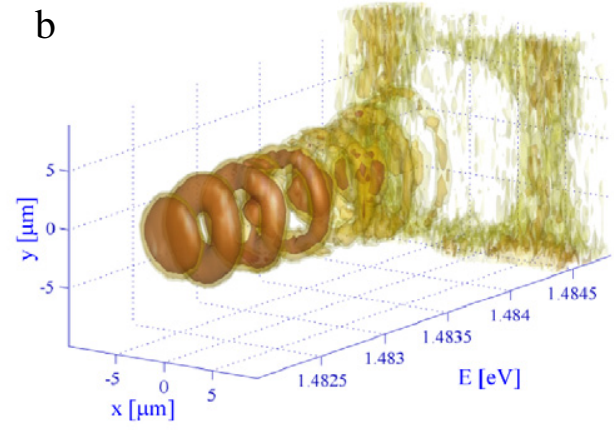

Fig. 2. $(x, y, E)$ view of the photoluminescence intensity of the system, for a mesa of (a) $3.5 \mu \mathrm{m}$ and (b) $9 \mu \mathrm{m}$ (only lower polariton states are shown). Isosurfaces of same intensity are plotted, allowing us to map the 2D spatial PD distribution of every confined and extended state for the upper and lower polaritons at the same time. For the sake of visibility, the energy scale is stretched on the lower polariton energy range. To observe easily all confined state PDs, the PL intensity at every energy was multiplied by a Boltzmann factor with $T=30 \mathrm{~K}$ for (a) and $T=70 \mathrm{~K}$ for (b), in order to compensate for the quasi thermal intensity distribution.

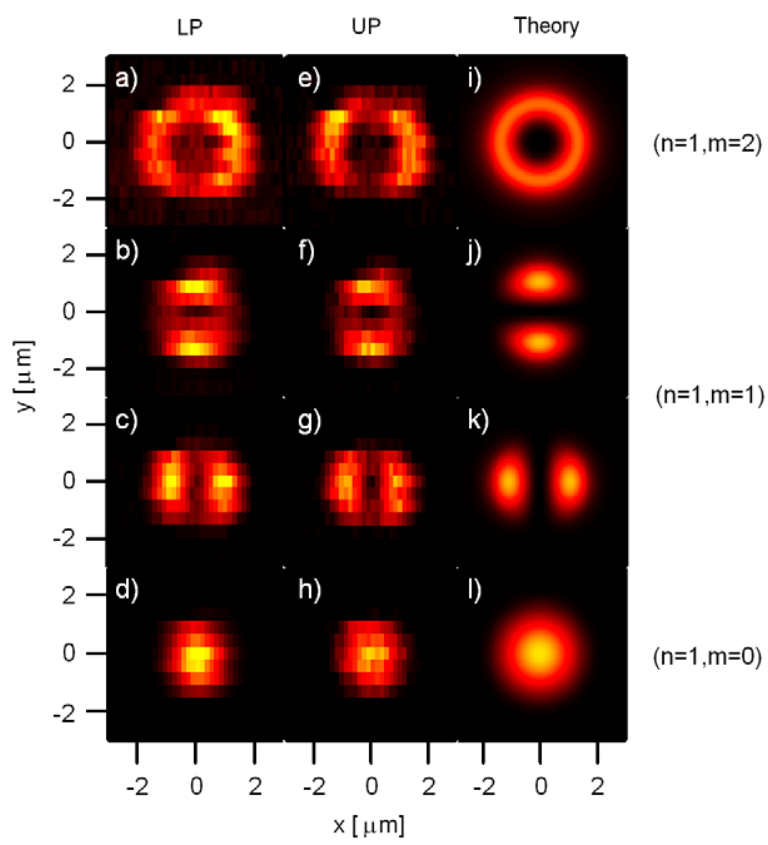

Fig. 3. Measured probability density patterns in a $3.5 \mu \mathrm{m}$ mesa, for lower polariton states (a)-(d), upper polariton states (e)-(h) and solutions of the Schrödinger equation for a cylindrical confinement (i)-(l), in a linear color scale. Parts (d), (h), (l) show a $(n=1, m=0)$ state pattern. Parts (b), (c), (f), (g), (j), (k) show two quasi-degenerate $(n=1, m=1)$ state patterns, the symmetry breaking being due to the elliptical shape of the mesa. Parts (a), (e), (i) show a $(n=1, m=2)$ state pattern for which the cylindrical symmetry is conserved, the symmetry breaking affecting only $m= \pm 1$ states.

the second spatial dimension. We obtain in this way a 3-dimensional mapping ( $x, y$, energy) of the PL intensity, which is presented in a 3D contour plot for the $3.5 \mu \mathrm{m}$ mesa in Fig. 2(a) and for the lower polariton of a $9 \mu \mathrm{m}$ mesa in Fig. 2(b). For the sake of visibility, we corrected the PL intensity at every energy by a Boltzmann factor, in order to counterbalance the thermalisation towards the lower energy 

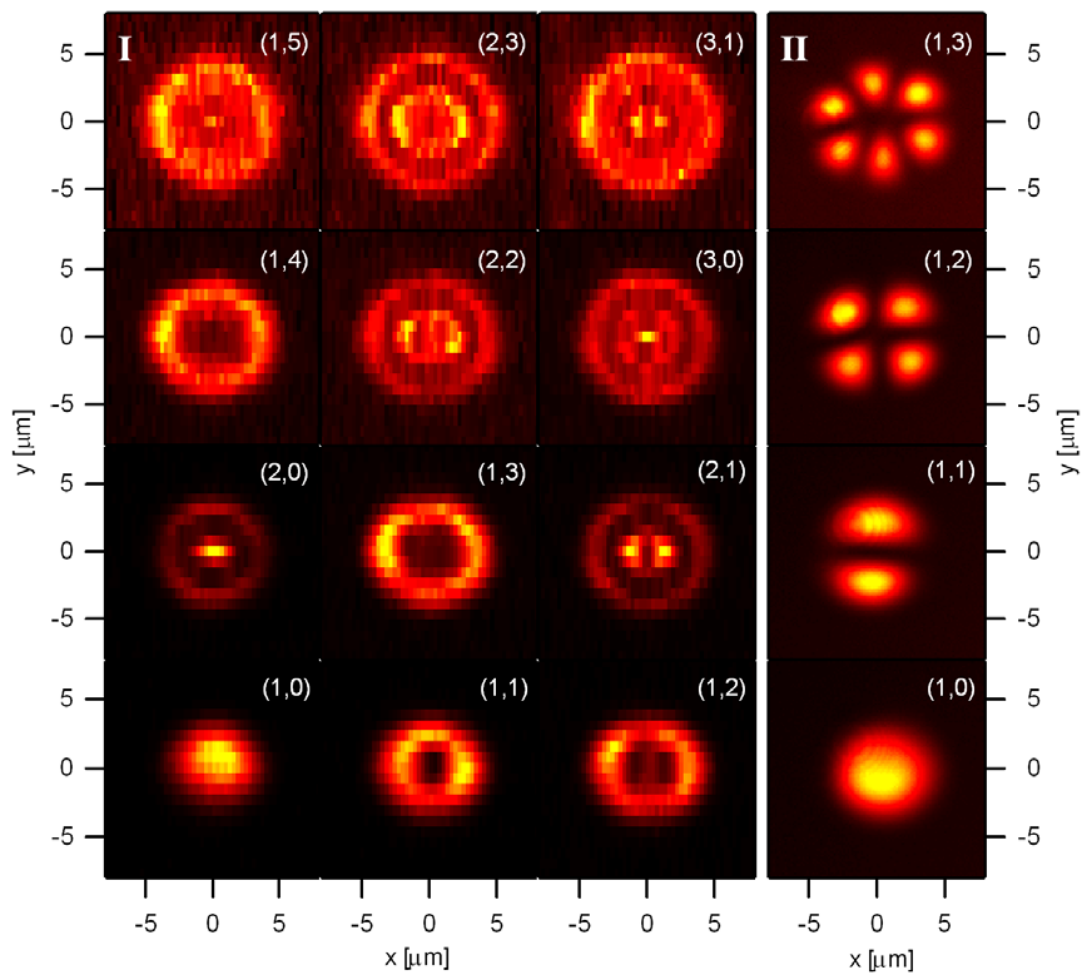

Fig. 4. Column I: Probability density patterns, measured using the same technique than in Fig. 3, for the lower polariton states in a $9 \mu \mathrm{m}$ mesa, in a linear color scale. Quantum numbers $(n, m)$ of the different confined states are indicated. All patterns show a cylindrical symmetry, except for the $(n=2, m=1)$ state, where $2 m=2$ lobes are distinguishable. Column II: Probability density patterns obtained by imaging the coherent emission of states confined in a $9 \mu$ m mesa, under resonant laser excitation of the state. The phase relationship between the $+m$ and $-m$ states is fixed by the laser, resulting in an interference pattern with $2 m$ lobes in the azimuthal direction.

states. The temperature of the system can be extracted from the PL data as described in Ref. [7]. It varies according to the excitation conditions and to the detuning of the photonic modes with respect to the excitonic resonance. On Fig. 2(a), all confined states of the upper and lower polaritons are visible, as well as the extended 2D polaritons, at a glance. For both the $3.5 \mu \mathrm{m}$ mesa and the $9 \mu \mathrm{m}$ mesa, one can clearly observe that the luminescence of the 2D polaritons vanishes at the exact position of the mesa. This feature is particularly meaningful for the lower 2D polariton, as it is nearly $100 \%$ excitonic at this position. It is a straightforward experimental demonstration that, although the confinement is initially acting on the photonic modes only, the excitonic component (and a fortiori the entire polariton) is eventually confined as well, due to the strong coupling between the light and the matter wave.

By cutting slices in the $(x, y)$ plane of this set of data we can retrieve 2D mappings of the probability distribution $\|\psi(r, \theta)\|^{2}$ of any eigenstate. This is plotted for the $3.5 \mu$ m mesa in Fig. 3 for the ground state and first two excited states of both the lower (Fig. 3(a)-(d)) and upper (Fig. 3(e)-(h)) polaritons. The bottom line of Fig. 3 shows a ground confined state pattern, with quantum numbers $(n=1, m=$ 0 ), as expected. Let us consider now the first excited state, exhibited on the two central lines of Fig. 3. We must point out here that in principle in a system with a perfect cylindrical symmetry, $\pm m$ states are degenerate with no fixed phase relation between each other. In time integrated measurement of PL intensity of the eigenstates, this symmetry prevents one from observing any azimuthal variation of the wavefunction. This is obviously not the case for the first excited state shown in Fig. 3(b), (c), (f) and (g) where one can observe a $(n=1, m=1)$ state pattern featuring two lobes, aligned along two orthogonal axes. This is an unambiguous sign of symmetry breaking, already observed spectrally 


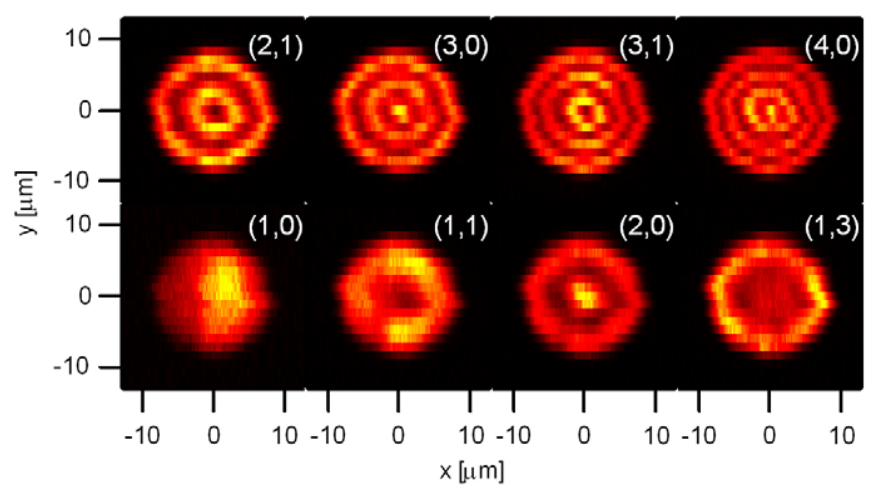

Fig. 5. Probability density patterns, measured using the same technique than in Fig. 3, for the lower polariton states in a $19 \mu \mathrm{m}$ mesa, in a linear color scale. Quantum numbers $(n, m)$ of the different confined states are indicated. For this confinement size, all confined states are cylindrically symmetric.

in Ref. [5], due to a slightly elliptical shape of the mesa. This gives rise to a lift of the degeneracy for the $m= \pm 1$ states $[3,4]$. The two split states then exhibit lobes which are pinned along the main axes of the elliptical confinement potential. This degeneracy lift only affects $m= \pm 1$ states, because the perturbation on the confinement potential scales as $\cos (2 \phi)$, where $\phi$ is the azimuthal angle. Indeed, for the ( $n=1, m=2)$ states displayed in Fig. 3(a) and (e), we do not observe lobes, neither a doublet structure.

Fig. 3(i)-(1) shows the corresponding solutions of the time-independent Schrödinger equation for a cylindrical confinement. Calculated patterns are similar for both upper and lower polaritons, as changing effective mass and confinement potential mainly changes eigen-energies. Excellent agreement can be observed with measured patterns. An azimuthal variation of the form $\mathrm{e}^{i m \phi}$ has been applied to Fig. 3(j) and $(\mathrm{k})$ in order to reproduce the effect of the symmetry breaking.

The ellipticity of the potential is much less pronounced for the $9 \mu \mathrm{m}$ mesa. One can indeed see in Fig. 2(b) and in Fig. 4, column I, that cylindrical symmetry is respected for almost all confined states. Only for the $(n=2, m=1)$ state pattern some lobes are distinguishable.

Eventually, Fig. 5 shows the state patterns obtained for a $19 \mu \mathrm{m}$ mesa. For this mesa size, the ellipticity is even less pronounced. This has for result that all confined states present a cylindrical symmetry.

It is however possible to break the symmetry by exciting resonantly the confined states [3]. In this case, the phase of the laser is imprinted to the $\pm m$ states, forcing the interference patterns to exhibit $2 m$ nodes. This is demonstrated in Fig. 4, column II, where the coherent emission pattern of several states of the $9 \mu \mathrm{m}$ mesa clearly feature lobes, although the emission patterns of these same states where cylindrically symmetric under non-resonant excitation.

In conclusion, we have performed a full ( $x, y$, energy) tomography of the photoluminescence of polariton states confined in cylindrical traps. This technique allows to retrieve $2 \mathrm{D}$ spatial probability density mappings for every eigenstate, for three different confinement sizes. In particular, these mappings have evidenced the effect of the elliptical geometry of the smaller traps through the breaking of the cylindrical symmetry on the azimuthal PD distribution for $m= \pm 1$ states. Probability density patterns are successfully compared with the solutions of the Schrödinger equation for a particle confined in a cylindrical trap. Eventually we demonstrate the breaking of the cylindrical symmetry by imprinting the phase of a resonant excitation laser, resulting in the formation of lobes in the real space probability density patterns.

\section{Acknowledgements}

We would like to thank K.G. Lagoudakis and M. Wouters for fruitful discussions. We acknowledge support from the Swiss National Research Foundation through the 'NCCR Quantum Photonics'. 


\section{References}

[1] C. Weisbuch, M. Nishioka, A. Ishikawa, Y. Arakawa, Phys. Rev. Lett. 69 (1992) 3314.

[2] R. Houdré, C. Weisbuch, R.P. Stanley, U. Oesterle, P. Pellandini, M. Ilegems, Phys. Rev. Lett. 73 (1994) 2043.

[3] R. Cerna, D. Sarchi, T.K. Paraïso, G. Nardin, Y. Léger, M. Richard, B. Pietka, O. El Daif, F. Morier-Genoud, V. Savona, M.T. Portella-Oberli, B. Deveaud-Plédran, arXiv:0904.4444v1.

[4] G. Nardin, T.K. Paraïso, R. Cerna, B. Pietka, Y. Léger, O. El Daif, F. Morier-Genoud, B. Deveaud-Plédran, Appl. Phys. Lett. 94 (2009).

[5] O. El Daif, A. Baas, T. Guillet, J.-P. Brantut, R. Idrissi Kaitouni, J.L. Staehli, F. Morier-Genoud, B. Deveaud, Appl. Phys. Lett. 88 (2006) 061105.

[6] A. Baas, O. El Daif, M. Richard, J.-P. Brantut, G. Nardin, R. Idrissi Kaitouni, T. Guillet, V. Savona, J.L. Staehli, F. Morier-Genoud, B. Deveaud, Phys. Status Solidi (b) 243 (2006) 2311.

[7] T.K. Paraïso, D. Sarchi, G. Nardin, R. Cerna, Y. Léger, B. Pietka, M. Richard, O. El Daif, F. Morier-Genoud, V. Savona, B. DeveaudPlédran, Phys. Rev. B 79 (2009) 045319.

[8] F. Tassone, C. Piermarocchi, V. Savona, A. Quattropani, P. Schwendimann, Phys. Rev. B 56 (1997) 7554.

[9] X. Leyronas, M. Combescot, Solid State Commun. 119 (2001) 631.

[10] The photonic and excitonic fractions are related to the detuning of the photonic mode with respect to the exciton energy. 